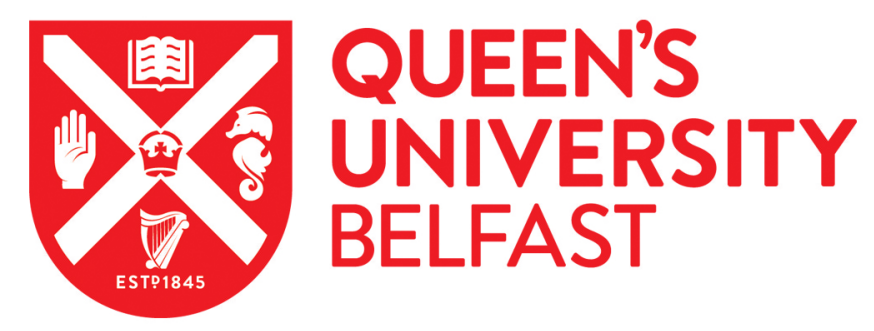

\title{
Numerical Investigation into the Mechanism Regarding the Inception and Evolution of Flow Unsteadiness Induced by Tip Leakage Flow in a Transonic Compressor
}

An, G., Wu, Y., Spence, S., Lang, J., Chen, Z., \& Mahmoudi, Y. (2020). Numerical Investigation into the Mechanism Regarding the Inception and Evolution of Flow Unsteadiness Induced by Tip Leakage Flow in a Transonic Compressor. Proceedings of the Institution of Mechanical Engineers, Part A: Journal of Power and Energy. https://doi.org/10.1177/0957650920910597

Published in:

Proceedings of the Institution of Mechanical Engineers, Part A: Journal of Power and Energy

Document Version:

Peer reviewed version

Queen's University Belfast - Research Portal:

Link to publication record in Queen's University Belfast Research Portal

\footnotetext{
Publisher rights

Copyright 2020 The Authors. This work is made available online in accordance with the publisher's policies. Please refer to any applicable terms of use of the publisher.
}

\section{General rights}

Copyright for the publications made accessible via the Queen's University Belfast Research Portal is retained by the author(s) and / or other copyright owners and it is a condition of accessing these publications that users recognise and abide by the legal requirements associated with these rights.

\section{Take down policy}

The Research Portal is Queen's institutional repository that provides access to Queen's research output. Every effort has been made to ensure that content in the Research Portal does not infringe any person's rights, or applicable UK laws. If you discover content in the Research Portal that you believe breaches copyright or violates any law, please contact openaccess@qub.ac.uk. 


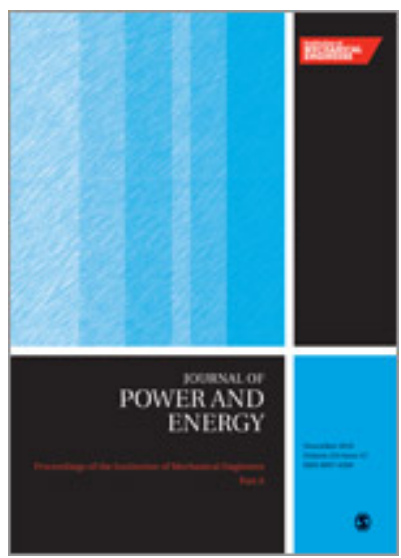

\section{Numerical Investigation into the Mechanism Regarding the Inception and Evolution of Flow Unsteadiness Induced by Tip Leakage Flow in a Transonic Compressor}

\begin{tabular}{|c|c|}
\hline Journal: & Part A: Journal of Power and Energy \\
\hline Manuscript ID & JPE-19-0384.R1 \\
\hline Manuscript Type: & Original Article \\
\hline $\begin{array}{r}\text { Date Submitted by the } \\
\text { Author: }\end{array}$ & 10-Dec-2019 \\
\hline Complete List of Authors: & $\begin{array}{l}\text { An, Guangyao; Northwestern Polytechnical University, School of Power } \\
\text { and Energy } \\
\text { Wu, Yanhui; Northwestern Polytechnical University, school of power and } \\
\text { energy } \\
\text { Spence, Stephen; Queen's University Belfast } \\
\text { Lang, Jinhua; Northwestern Polytechnical University } \\
\text { Chen, Zhiyang; Northwestern Polytechnical University } \\
\text { Larimi, Yasser; Queen's University Belfast }\end{array}$ \\
\hline Keywords: & $\begin{array}{l}\text { Compressor aerodynamics, Axial flow compressors, Flow Unsteadiness, } \\
\text { Tip Leakage Flow }\end{array}$ \\
\hline Abstract: & $\begin{array}{l}\text { Unsteady flow in the blade tip region of modern axial flow compressors is } \\
\text { one of the sources of loss, noise and blade vibration. In some cases, it is } \\
\text { potentially linked to stall inception. } \\
\text { In this paper, the complex flow fields in the blade tip region of a } \\
\text { transonic axial flow compressor rotor have been numerically } \\
\text { investigated. The predicted results were validated by experimental data. } \\
\text { Analyses of monitoring results of numerical probes showed that three } \\
\text { typical flow characteristics occurred as the operating condition } \\
\text { approached the stability limit: no flow fluctuation at the first operating } \\
\text { point; flow fluctuation with high frequency and low amplitude at the } \\
\text { second operating point; flow fluctuation with low frequency and high } \\
\text { amplitude at the third operating point. } \\
\text { Further analysis of the tip flow field showed that the evolution of the tip } \\
\text { leakage vortex (TLV) experienced three stages as the rotor was } \\
\text { throttled. At the first stage, the TLV did not breakdown. At the second } \\
\text { stage, a bubble-type breakdown of the TLV occurred. At the third stage, } \\
\text { a spiral-type breakdown of TLV occurred. }\end{array}$ \\
\hline
\end{tabular}


The current study demonstrated that the flow unsteadiness that appears within the test rotor was induced by the TLV breakdown. Furthermore, with the transformation of the vortex breakdown form, the characteristic frequency and amplitude of the flow oscillation substantially changed.

\section{SCHOLARONE ${ }^{m}$ \\ Manuscripts}




\title{
Numerical Investigation into the Mechanism Regarding the
}

\section{Inception and Evolution of Flow Unsteadiness Induced by Tip Leakage Flow in a Transonic Compressor}

\author{
Guangyao $\mathrm{An}^{1}$, Yanhui $\mathrm{Wu}^{1} \uparrow$, Stephen Spence ${ }^{2}$, Jinhua Lang ${ }^{1}$, Zhiyang Chen ${ }^{1}$, Yasser Mahmoudi Larimi ${ }^{2}$ \\ ${ }^{1}$ School of Power and Energy, Northwestern Polytechnical University, Xi'an, Shaanxi, 710129, China \\ ${ }^{2}$ School of Mechanical and Aerospace Engineering, Queen's University Belfast, Belfast BT9 5AH, UK \\ †Corresponding Author Email: wyh@nwpu.edu.cn
}

\begin{abstract}
Unsteady flow in the blade tip region of modern axial flow compressors is one of the sources of loss, noise and blade vibration. In some cases, it is potentially linked to stall inception.

In this paper, the complex flow fields in the blade tip region of a transonic axial flow compressor rotor have been numerically investigated. The predicted results were validated by experimental data.

Analyses of monitoring results of numerical probes showed that three typical flow characteristics occurred as the operating condition approached the stability limit: no flow fluctuation at the first operating point; flow fluctuation with high frequency and low amplitude at the second operating point; flow fluctuation with low frequency and high amplitude at the third operating point.
\end{abstract}


Further analysis of the tip flow field showed that the evolution of the tip leakage vortex (TLV) experienced three stages as the rotor was throttled. At the first stage, the TLV did not breakdown. At the second stage, a bubble-type breakdown of the TLV occurred. At the third stage, a spiral-type breakdown of TLV occurred.

The current study demonstrated that the flow unsteadiness that appears within the test rotor was induced by the TLV breakdown. Furthermore, with the transformation of the vortex breakdown form, the characteristic frequency and amplitude of the flow oscillation substantially changed.

Key words: Flow unsteadiness; Tip leakage vortex; Vortex breakdown; Transonic compressor rotor.

\section{List of Abbreviations}

\begin{tabular}{llll} 
TLV & Tip Leakage Vortex & CAL & Calculation \\
TSV & Tip Secondary Vortex & PS & Pressure Side \\
\multirow{2}{*}{ URANS } & Unsteady Reynolds-averaged & SS & Suction Side \\
& Navier-Stokes & & \\
LE & Leading Edge & FFT & Fast Fourier Transformation \\
TE & Trailing Edge & BPF & Blade Passing Frequency \\
EXP & Experiment & Vol & Volume \\
ref & Reference & TAV & Time Averaged
\end{tabular}




\section{List of Symbols}

\begin{tabular}{|c|c|c|c|}
\hline$C_{a x}$ & $\begin{array}{l}\text { Axial chord length at the chosen } \\
\text { spanwise location }\end{array}$ & $H$ & Helicity \\
\hline$m$ & Mass flow rate & $\zeta$ & $\begin{array}{l}\text { Streamwise component of } \\
\text { normalised vorticity }\end{array}$ \\
\hline$\pi$ & Total pressure ratio & $\xi$ & $\begin{array}{l}\text { Radial component of normalised } \\
\text { vorticity }\end{array}$ \\
\hline$V_{t}$ & Relative tangential velocity & $\eta$ & $\begin{array}{l}\text { Circumferential component of } \\
\text { normalised vorticity }\end{array}$ \\
\hline$f$ & Frequency & $W$ & Relative velocity \\
\hline$A_{m}$ & Amplitude of mass flow rate & $P$ & Static pressure \\
\hline$A_{p}$ & Amplitude of static pressure & $\tau$ & Physical time step \\
\hline$\omega$ & Normalized absolute vorticity & $C_{p}$ & Static pressure coefficient \\
\hline$\Omega$ & Angular velocity at rotor tip & $t$ & Non-dimensional time \\
\hline$\rho$ & Density & $U$ & Blade tip speed \\
\hline
\end{tabular}

\subsection{INTRODUCTION}

Tip leakage flow through the radial clearance between the blade tip and the casing has gained extensive attention by researchers in the turbomachinery field due to the fact that it contributes a significant portion of the overall loss [1] and has significant effects on the performance and stability of compressors [2-3]. The tip clearance flow field is characterized by a streamwise slender vortex, i.e. the so-called tip leakage vortex (TLV) which is generated by the rolling-up of the shear layer between leakage jet and the main 
flow [4]. Recent investigations indicated that the development of the tip leakage vortex not only generated loss but also induces strong unsteady flow which has been determined to be the main cause of tip noise [5] and blade vibration [6], and severely affects the stability margin of compressors.

Due to its great impacts on compressors, flow unsteadiness has drawn the attention of a large number of researchers [7-19]. The knowledge reported to date allows the flow unsteadiness to be divided into three categories; TLV breakdown, shock interaction and self-induced unsteadiness. Each of these will now be discussed.

(1) TLV breakdown. Given that the blade tip region is characterized by a high adverse pressure gradient, the TLV tends to break down under certain circumstances. In as much as the authors has been able to determine, the phenomenon of TLV breakdown was first reported by Schlechtriem [7] who conducted numerical simulations on a transonic compressor rotor. He suggested that the inherent unsteady modes of the TLV breakdown are responsible for upstream propagating pressure waves which cause further unsteady pressure fluctuations. Furukawa et al. [8-9] also observed the TLV breakdown phenomenon in a low-speed axial compressor rotor at the near stall condition. They pointed out that the TLV breakdown gives rise to a self-sustained flow oscillation that induces a cyclic variation in the pressure on the pressure side near the tip. Later on, Yamada et al. [10-11] performed single passage simulations on a transonic compressor rotor. The results showed a spiral-type TLV breakdown due to the interaction between the TLV and the shock and that the characteristic frequency of the TLV breakdown induced flow oscillation corresponds to $60 \%$ blade passing frequency (BPF). 
(2) Shock oscillation and TLV/shock interaction. Under certain circumstances, for example in Hah's investigation [12], no TLV breakdown was observed since the TLV was found to be at an oblique angle to the shock and the swirl intensity of the vortex core was too weak for the vortex breakdown. Even so, the flow field became unsteady due to the shock oscillation [12]. Further experimental study indicated that the interaction between the TLV and the shock was an inherently unsteady phenomenon in the compressor system and contributed more effects to the flow field unsteadiness than just the shock oscillation [13].

(3) Self-induced unsteadiness. Zhang et al. [14-15] proposed a different mechanism for the origin of flow unsteadiness. They suggested that the blade loading dominated the flow oscillation in the tip region. Specifically, the periodic flow oscillation was a result of a dynamic balance between the loading and unloading of the blade. Their conclusions were then verified by Tong et al. [16] through experimental approaches. Tong et al. [16] called it self-induced unsteadiness, since it was essentially a result of the alternative action of the main flow and the tip leakage flow, which caused a loading and unloading of the blade. Another step forward on this topic was made by Du et al. [17], who proposed a correlation factor which could be used as an indicator for the onset of the self-induced flow unsteadiness.

(4) The formation and development of the tip secondary vortex (TSV). Wu et al. [18] found a new vortex structure that originated from the TLV breakdown region. This new vortex structure was designated as the tip secondary vortex (TSV). They concluded that the cyclical motion of the TSV is the main source of the periodic variation of the tip flow field. Subsequent experiments [19] performed on the same rig clearly captured the structure 
of the TSV and revealed that the interaction between the broken-down TLV and the lowenergy fluid from adjacent passage accounted for the formation of the TSV.

In light of the research discussed above, it is apparent that tip leakage flow is closely related to the flow unsteadiness. Although opinions concerning the origins and the underlying flow mechanism of flow unsteadiness on different compressors vary widely duo to the differences in their design parameters, it is well accepted that the TLV breakdown has great impact on the tip flow field and causes flow unsteadiness. However, no detailed study has been conducted to reveal the origin and mechanism of flow unsteadiness induced by TLV breakdown. It is the motivation of the current study.

In this paper, a series of URANS simulations were carried out on a transonic compressor rotor, NASA Rotor 35. Section 2 describes the numerical methodology employed in this study. In section 3.1, the performance and the predicted flow fields were evaluated against experimental data. In section 3.2, a series of Fast Fourier Transformation (FFT) analyses were conducted to show the characteristic frequencies and amplitudes of flow unsteadiness. In section 3.3 and 3.4, the flow fields before and after the inception of flow unsteadiness were investigated in detail. The primary objective of current study was to elucidate some insights into the underlying mechanism of flow unsteadiness. 


\section{NUMERICAL METHODOLOGY}

\subsection{Transonic compressor rotor}

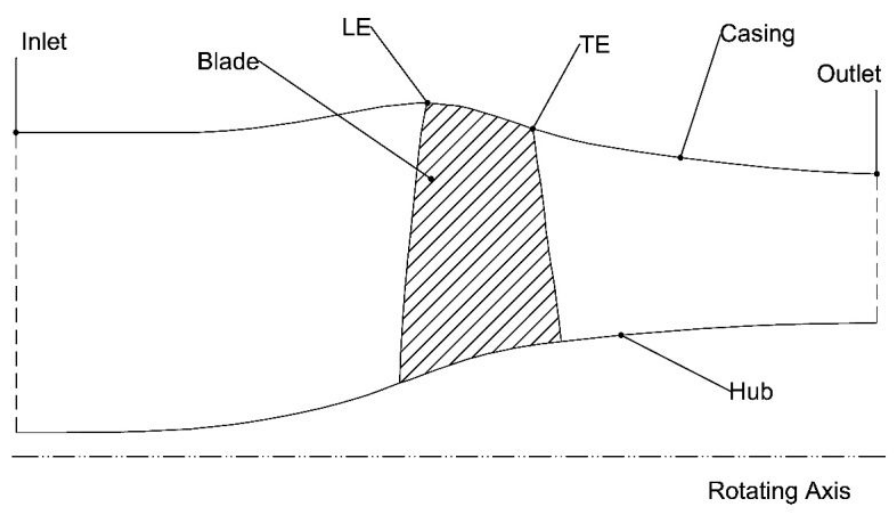

Figure 1 A meridional view of the rotor passage

The study was performed on NASA Rotor 35, a transonic axial compressor rotor, which was originally designed as an inlet rotor for a multi-stage compressor and tested at NASA Lewis Research Centre with a design speed of 17188.17 RPM. The rotor had 36 blades, an inlet tip radius of $254 \mathrm{~mm}$, a hub-tip radius ratio of 0.70 , an aspect ratio of 1.19 , a tip solidity of 1.3 , and an axial chord of $27.2 \mathrm{~mm}$ at the tip and $41.2 \mathrm{~mm}$ at the hub. The tip clearance height was $0.74 \mathrm{~mm}$. The spanwise location of the tip of the blade at leading edge (LE) was $99.5 \%$. The compressor was designed for axial inlet flow and the inlet relative velocity was $454.5 \mathrm{~m} / \mathrm{s}$ at blade tip at the design speed. To help better understand the structure, a meridional view of the rotor passage is displayed in Figure 1. Detailed design parameters can be found in the report of Reid and Moore [20]. Experimental data and the time-averaged flow field were measured by Van Zante [21] using pneumatic probes and a Laser Doppler Velocimetry system, which are available to validate numerical simulations. 


\subsection{Computational domain}

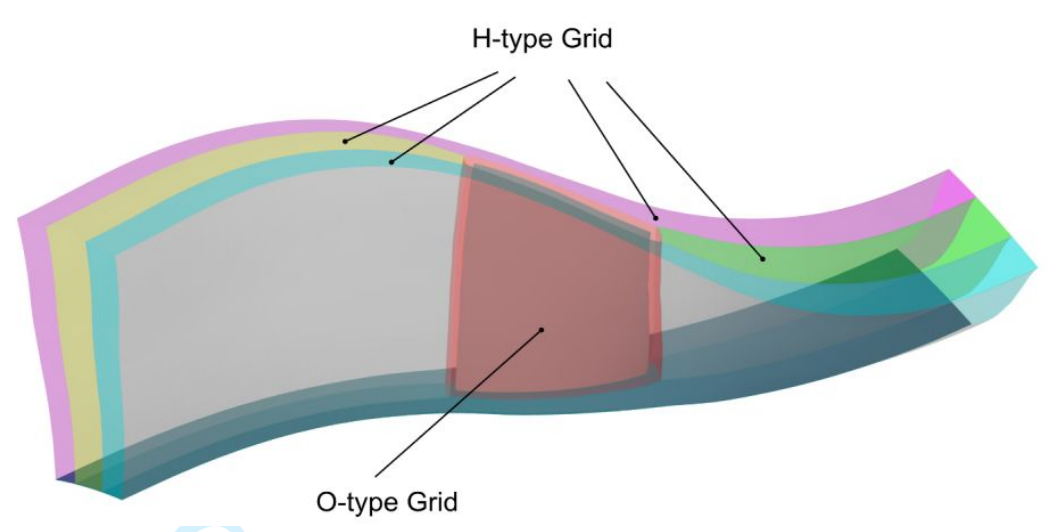

Figure 2 Topology of the main flow region

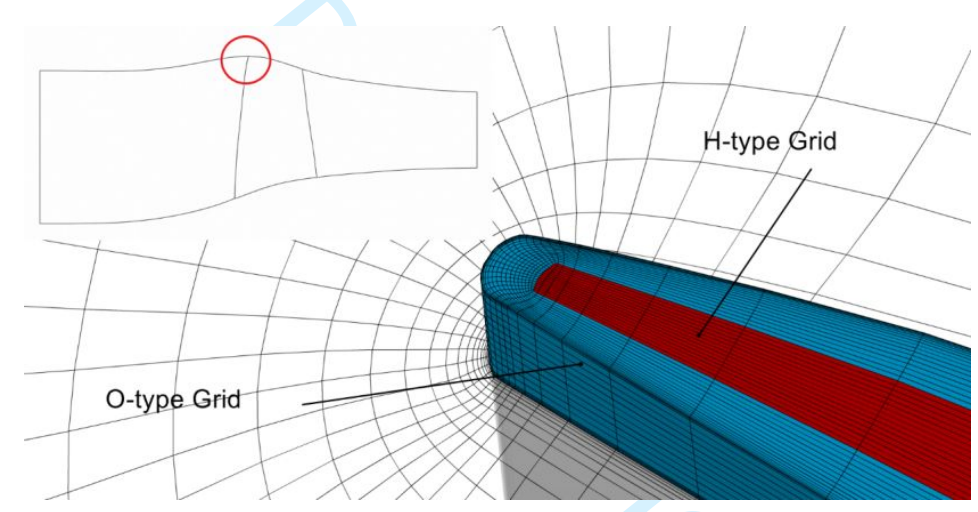

Figure 3 Butterfly topology at the tip flow region

For the sake of reducing computational efforts, the domain was truncated to a single passage. The computational grid employed in this study is shown in Figure 2 and Figure 3. The blade-passage domain has two zones: the main flow region and the tip flow region. In light of the investigation of Van Zante et al. [21], grids near the casing were refined so as to achieve accurate numerical simulation of the tip clearance flow. The mesh was generated using AutoGrid 5 (NUMECA INTERNATIONAL). A grid convergence study was performed to ensure that the solution was independent of the grid setup. Three grids of different densities $\left(1.5 \times 10^{6}, 2.4 \times 10^{6}\right.$ and $\left.3.3 \times 10^{6}\right)$ were tested. Performance curves of 
different grid topologies were compared with the experimental data. Based on the results, the medium grid was adopted in the study since the predicted performance curve agreed quite good with the experimental data. A further increase of the grid density will introduce small improvements of the simulation but extra computing time. The main flow region was subdivided into 5 grid blocks: an O-type grid was generated around the blade with 53 cells in the pitchwise direction, 97 cells in the spanwise direction and 217 cells in the streamwise direction; the others were all H-type grids. Butterfly topology was used to model the tip gap region (see Fig. 3): an H-type grid was generated in the inner zone, which consisted of 25 cells in the pitchwise direction, 37 cells in the spanwise direction, and 127 cells in the streamwise direction, while an O-type grid was generated in the outer zone, which consist of $25 \times 37 \times 127$ cells in the pitchwise, spanwise, and streamwise directions, respectively. The cells near the solid walls were refined and gave $y+$ value approximately equal to 1 along solid walls.

\subsection{Arrangement scheme for numerical probes}

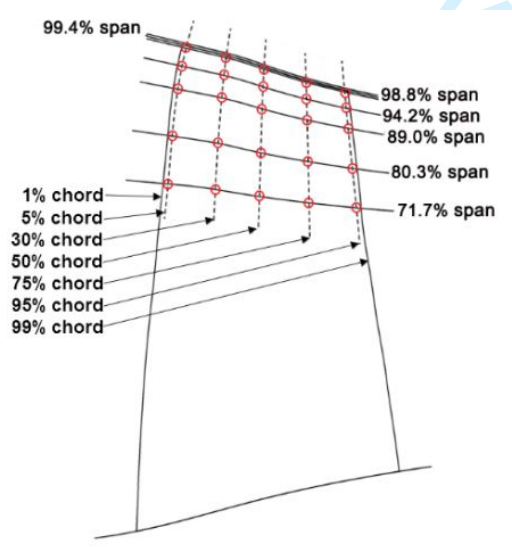

Figure 4 Location of numerical probes on the pressure surface 
Figure 4 shows the locations of a series of numerical probes used to analyse the results. A previous study on the same rotor by $\mathrm{Wu}$ and $\mathrm{An}$ [22] showed that the most prominent unsteady region was located on the pressure surface near the blade tip. Therefore, in the current study the numerical probes were arranged on the pressure surface and above $70 \%$ of blade span. As can be seen in Figure 4, the spanwise locations of probes were $99.4 \%$, $98.8 \%, 94.2 \%, 89.0 \%, 80.3 \%$, and $71.7 \%$ of blade span respectively. With the leading edge chosen as a datum reference, the axial locations of probes were $1 \%, 5 \%, 30 \%, 50 \%$, $75 \%, 90 \%, 99 \%$ of $C_{a x}$

\subsection{Boundary conditions}

No-slip and adiabatic conditions were imposed at the solid walls. The flow was assumed to be steady at both the inlet and outlet of the CFD domain. Inlet flow direction (axial direction), total pressure $(101325 \mathrm{~Pa})$ and total temperature $(288 \mathrm{~K})$ were specified at the inlet surface. Averaged static pressure was specified at the outlet of computational domain. The operating point of the compressor was changed by varying the outlet static pressure.

\subsection{Numerical scheme}

The present simulations were performed with a commercial flow solver EURANUS which was developed by NUMECA. The three-dimensional Reynolds-averaged NavierStokes equations were discretized in space using a cell-centered finite volume formulation. A second-order upwind scheme based on a flux difference splitting formulation was chosen 
to evaluate the inviscid fluxes so as to capture the tip leakage vortex sharply near the endwall. The viscous fluxes were determined in a central differencing manner with Gauss's theorem. An explicit Runge-Kutta scheme with local time step was used to obtain the steady solutions, while the unsteady solutions were obtained by an implicit dual timestepping method proposed by Jameson [23]. A low Reynolds number k-epsilon turbulence model (low Re Yang-Shih) was chosen to estimate the eddy viscosity.

The physical time step for time-accurate simulations was determined by the rotational speed, and the time for each rotor blade pitch to pass was divided into 30 physical time steps (3.23208e-6s for each time step). Within each physical time step, 50 pseudo time iterations were performed. During the development of the numerical model the authors tested 4 different set-ups (20/30/40/50 time steps per pitch), which showed that the selected physical time step scale of 30 steps per pitch was sufficient to capture the details of the flow field.

The convergence criteria used in the steady simulations were as follows: mass flow difference at the inlet and outlet $<0.01 \mathrm{~kg} / \mathrm{s}$, total-to-total isentropic efficiency fluctuation $<0.05 \%$. In unsteady simulations, the results were recorded after the pressure fluctuation of the numerical probes showed periodicity. 


\section{RESULTS AND DISCUSSIONS}

\subsection{Validation of numerical simulation}

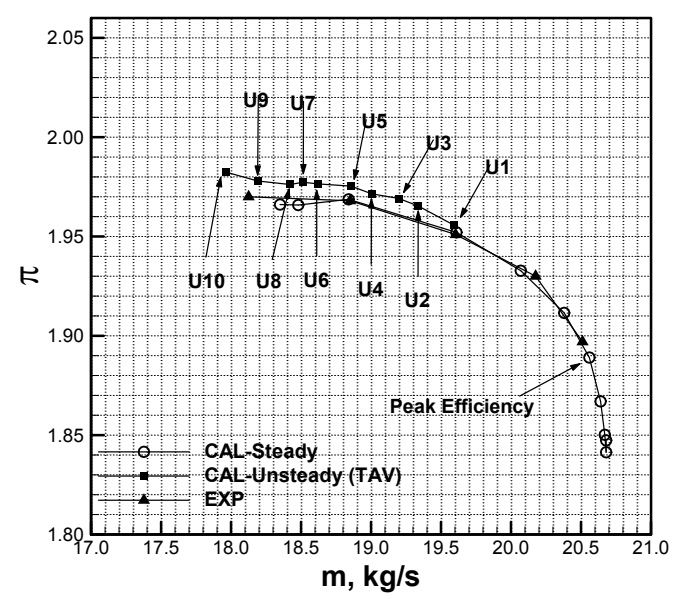

Figure 5 Total pressure ratio characteristic

Figure 5 shows a comparison of the predicted and measured total pressure ratio curves at the design speed. Initially, a series of steady simulations were conducted. Then 10 unsteady simulations were performed based on the steady results. Note that the unsteady results shown in Fig. 5 were time averaged values. As can be seen, the unsteady predicted total pressure ratio curve matched the experimental data well, but it was slightly above the experimental flow rate (about $0.6 \%$ ) as the stall limit was approached. The largest difference of $0.164 \mathrm{~kg} / \mathrm{s}$ between the predicted and experimental mass flow rate occurred near the stall limit; the measurement uncertainties in the experiment conducted by Van Zante [21] were stated as $\pm 0.3 \mathrm{~kg} / \mathrm{s}$. Thus, the predicted mass flow rate was within a satisfactory range. 


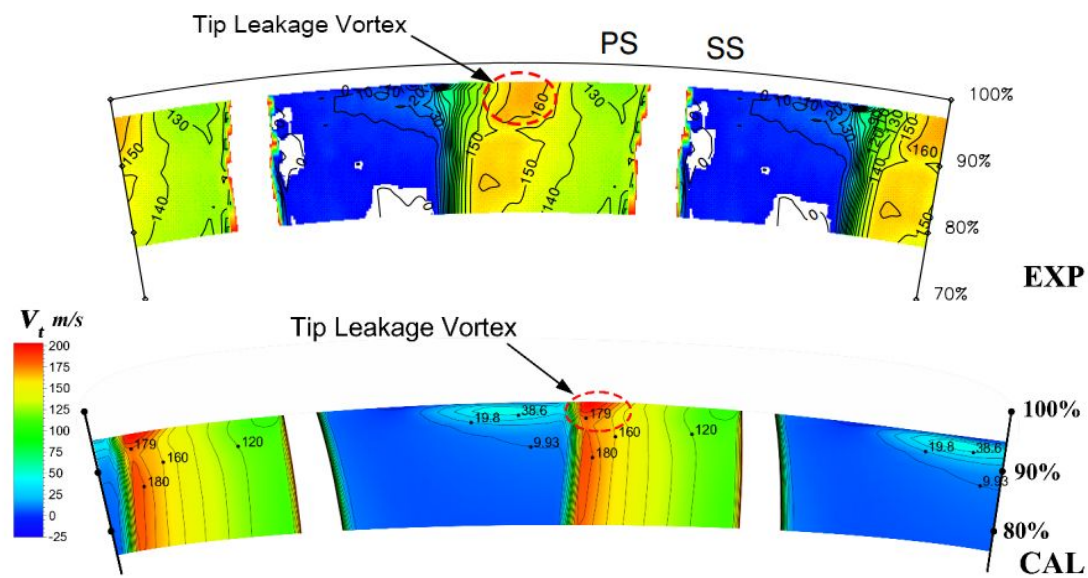

(a) $33 \%$ of $C_{a x}$

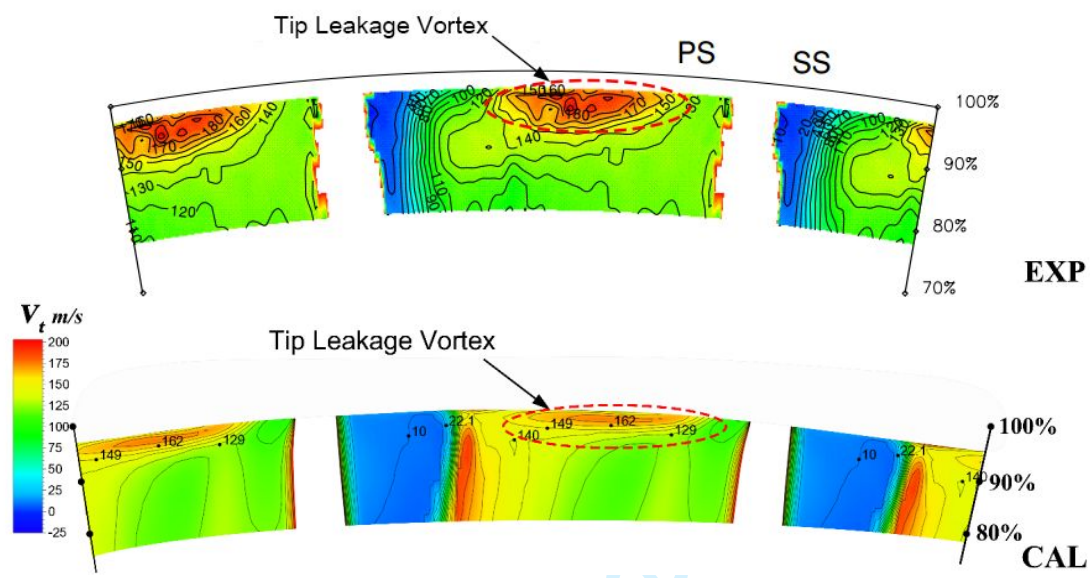

(b) $55 \%$ of $C_{a x}$

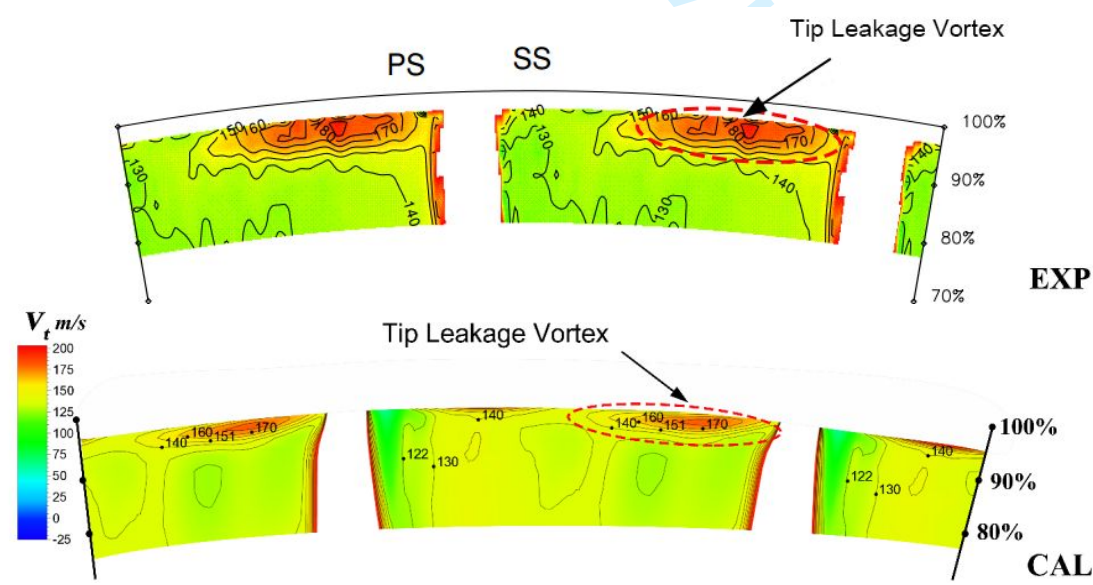

(c) $72 \%$ of $C_{a x}$

Figure 6 The measured and predicted tangential velocity distributions on cross channels at the peak-efficiency condition 
In Figure 6, The absolute tangential velocity distributions on cross channels at $33 \%$, $53 \%$ and $72 \%$ rotor chord are compared between numerical and experimental results at the peak-efficiency condition. It should be noted that, in this paper where the authors mainly focus on the behaviour of the TLV, the validation of the strength and extent of the TLV is the main consideration. As can be seen in Fig. 6, relatively high velocity regions which represent the TLV are well predicted at each axial location. Moreover, the absolute values of tangential velocity associated with the TLV match the experimental velocities well.

On the whole, the results presented in Figs. 5 and 6 show that the current simulations produce a satisfactory comparison with the available measurement data, thus providing confidence in further analysis of the computed flow fields.

\subsection{Analyses of monitoring results of numerical probes}

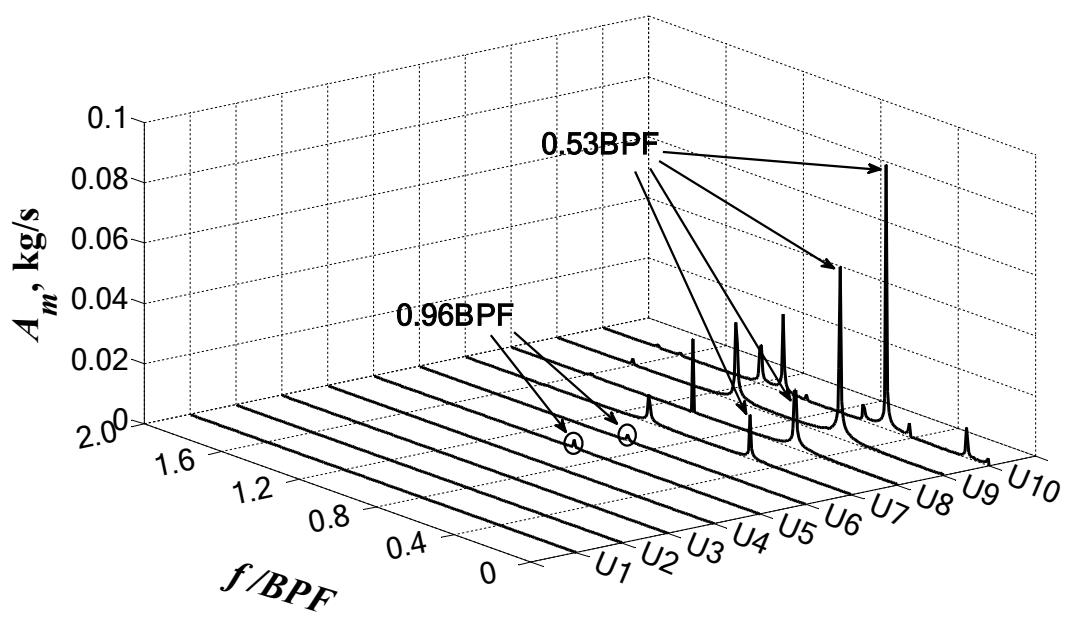

Figure 7 Frequencies and amplitudes of fluctuations in the outlet mass flow rate for condition U1-U10 
Figure 7 shows frequencies and amplitudes of fluctuations in the outlet mass flow rate under the 8 different operating conditions labelled in Fig. 5. Three typical flow characteristics can be observed:

(1) As the operating condition approached the stability limit, flow oscillation was first observed at U5.

(2) In general, two different groups of outlet mass flow oscillations were apparent at different conditions, which can be divided according to their frequency. Outlet mass flow oscillations first appear at U5 and U6 with a characteristic frequency approximately equal to the blade passing frequency (BPF). A second outlet mass flow oscillations appears from U7 to U10 with a larger amplitude and a lower characteristic frequency of approximately half of the BPF.

(3) The frequency of the outlet mass flow oscillation reduces almost by half (from 0.96BPF to $0.53 \mathrm{BPF}$ ) and the amplitude jumps significantly during the transition from U6 to U7.

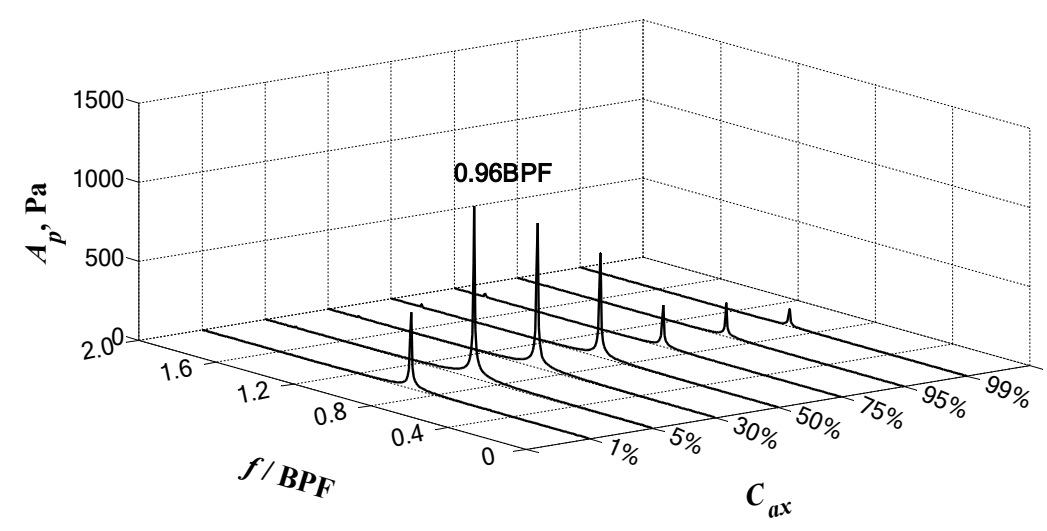

(a) Probes at $98.8 \%$ span 


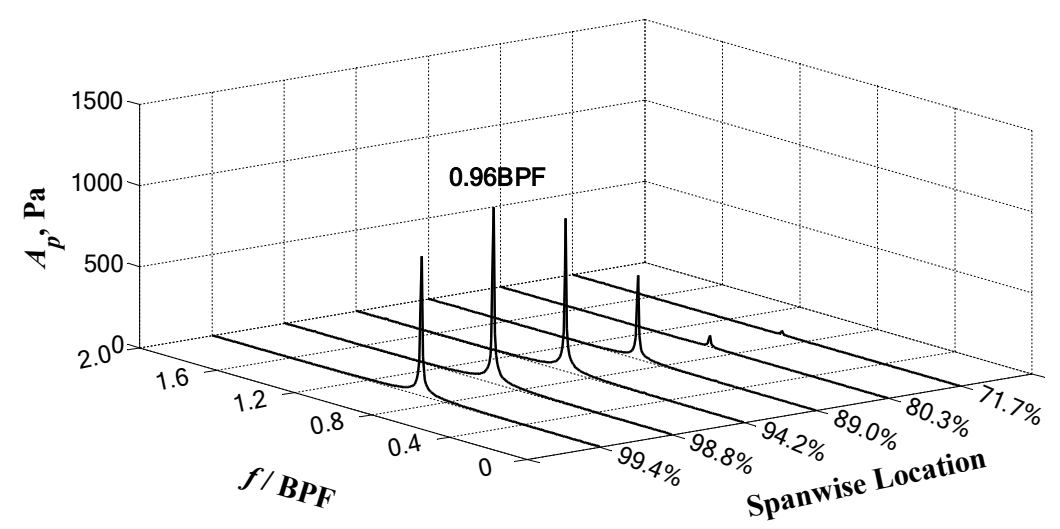

(b) Probes at $5 \% C_{a x}$

Figure 8 FFT spectrum of fluctuations in static pressure from the numerical probes for condition U5

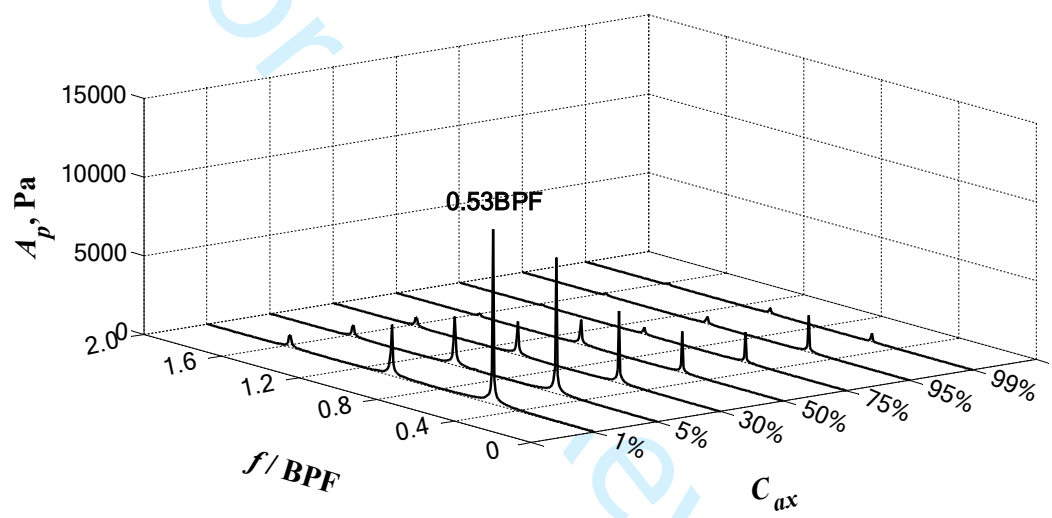

(a) Probes at $98.8 \%$ span

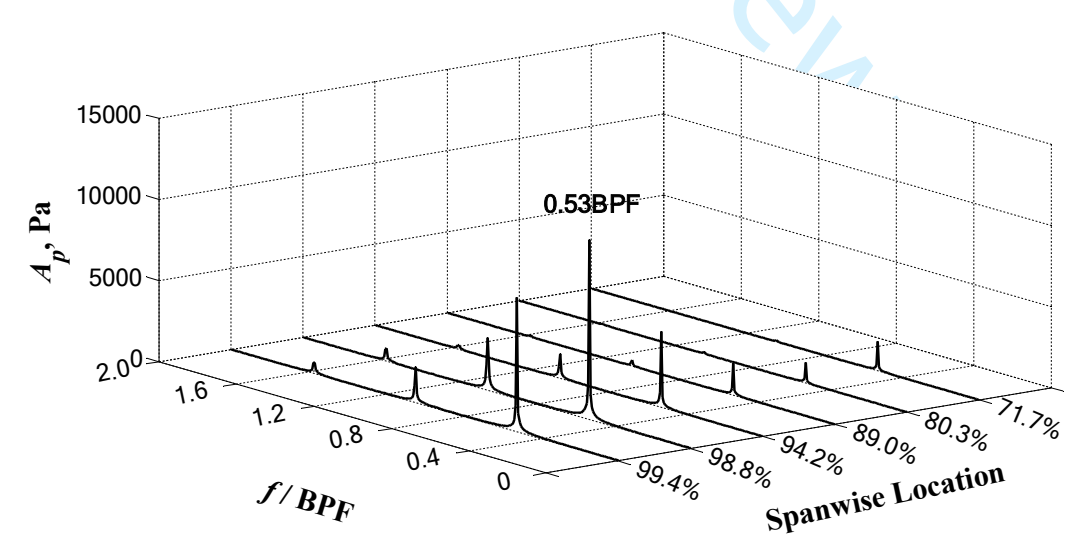

(b) Probes at $1 \% C_{a x}$

Figure 9 FFT spectrum of fluctuations in static pressure from the numerical probes for condition U7 
Figures 8 and 9 present the FFT results of sampling data from the numerical probes on the pressure surface that were defined in Fig. 4 for two characteristic conditions where flow unsteadiness was detected. The results show that the maximum amplitudes of the pressure oscillations at both conditions appear at the leading edge of the blade tip. Two distinct sources of pressure unsteadiness are also seen in Fig. 9 for the U7 condition. The results show that the unsteadiness is concentrated in the higher span region and in the first part of the chord. Furthermore, the frequencies of the pressure oscillations presented in Figs. 8 and 9 correspond to the frequencies of the outlet mass flow oscillations shown in Figure 7. This indicates that the tip flow field dominates the flow unsteadiness of the whole system. Thus, it is reasonable to investigate the origin of flow unsteadiness through analysing the flow characteristics near the leading edge of the blade tip region.

For the convenience of investigating the origin and mechanisms of the unsteady behaviour of tip flow field, further analyses and discussion in this paper are divided into two parts. In the first part, flow details before the inception of tip flow unsteadiness are presented. In the second part, flow details after the appearance of flow unsteadiness are analysed and discussed. 


\subsection{Flow details before the inception of tip flow unsteadiness}

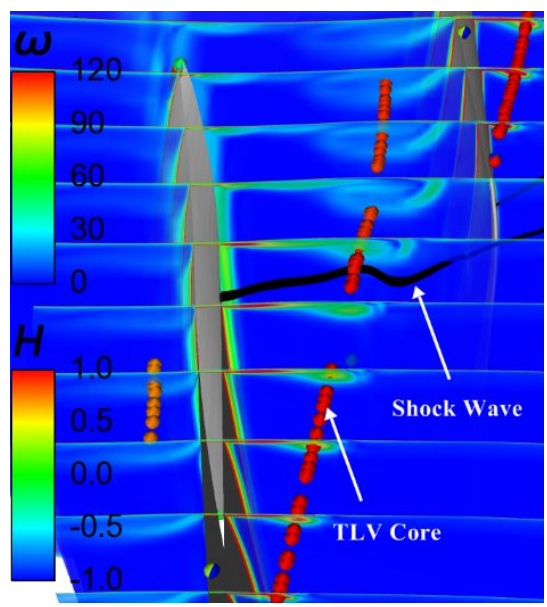

(a) U1 Condition

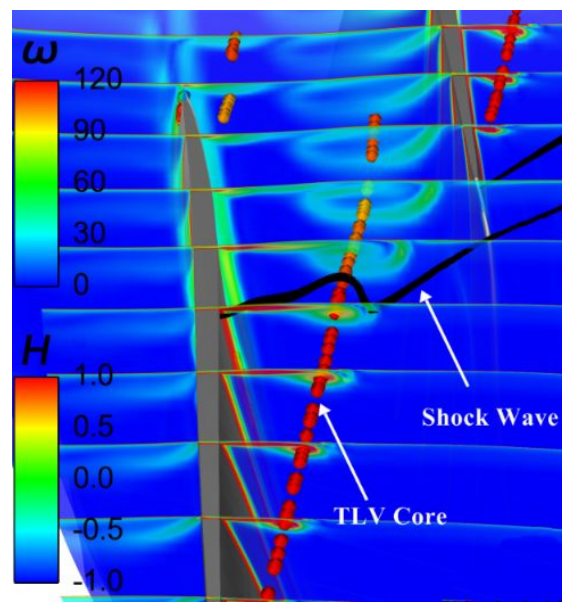

(b) U4 Condition

Figure 10 Instantaneous normalized absolute vorticity distributions on cross-channel planes and TLV core coloured with helicity

Figure 10 shows the configuration of TLV indicated by vorticity contours and the location of the vortex core has been extracted by eigenvector method which was proposed by Sujudi et al.[24]. Contour plots of normalized absolute vorticity on different crosschannel planes are presented here to help characterize the development of the TLV when growing downstream. These cuts are approximately perpendicular to the trajectory of the TLV. Note that the absolute vorticity was normalised by $2 \Omega$, where $\Omega$ is the angular velocity of at the rotor tip. Helicity was defined as follows: $H=\frac{\vec{\omega} \cdot \vec{W}}{|\vec{\omega}| \cdot \mid \vec{W}}$, where $\omega$ is the normalised vorticity and $W$ is the relative velocity. It represents the angle between the vorticity and the relative velocity.

It was found that the TLV core extracted by the eigenvector method (coloured balls) accurately showed the trajectory of the TLV, which justified the validity of this method. The vorticity distribution on cross-channel planes shows that the TLV has a region of 
concentrated vorticity upstream of the shock wave. Downstream of the shock wave, this vorticity-concentrated region disappears, accompanied by an expansion of the vortex core region. This is because of the interaction between the vortex and the shock wave. Specifically, to satisfy conservation of mass flow, when the axial velocity of the vortex core decreases suddenly as the vortex crosses the shock wave, an outflow in the perpendicular plane is then required, resulting in an increase of the vortex diameter. Although the vorticity distribution in this operating condition resembles that of vortex breakdown, the TLV does not actually breakdown at these two operating conditions of U1 and U4. This is determined by considering the velocity distribution along the vortex core (coloured by helicity): reverse flow, which is one of the criteria of vortex breakdown, is not observed along the vortex core line.

It is also observed that the region of expanding vorticity after the shock becomes more extensive as the flow rate decreases from U1 to U4, and it gradually moves to the pressure side of the blade.

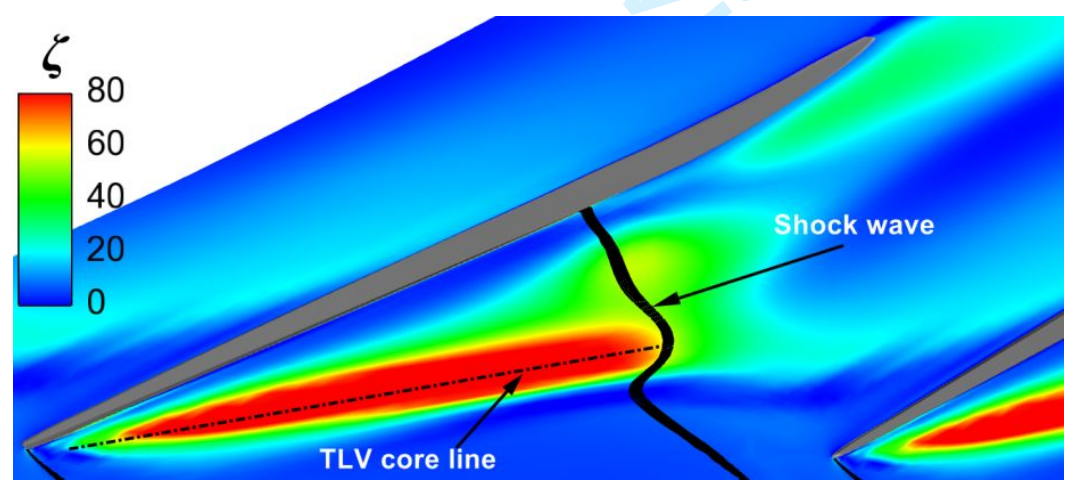

Figure 11 Streamwise component of normalised vorticity distribution at $98 \%$ span at $\mathrm{U} 4$

Figure 11 presents the streamwise component of normalised vorticity distribution on the blade-to-blade plane. It is found the streamwise component of vorticity in the TLV core 
region decreases significantly when crossing the shock wave. As previously noted, the vortex core experiences an expansion after the shock wave. According to the conservation of angular momentum, an increase of the vortex diameter would cause a decrease of the angular velocity, resulting in a decrease of the streamwise component of vorticity.

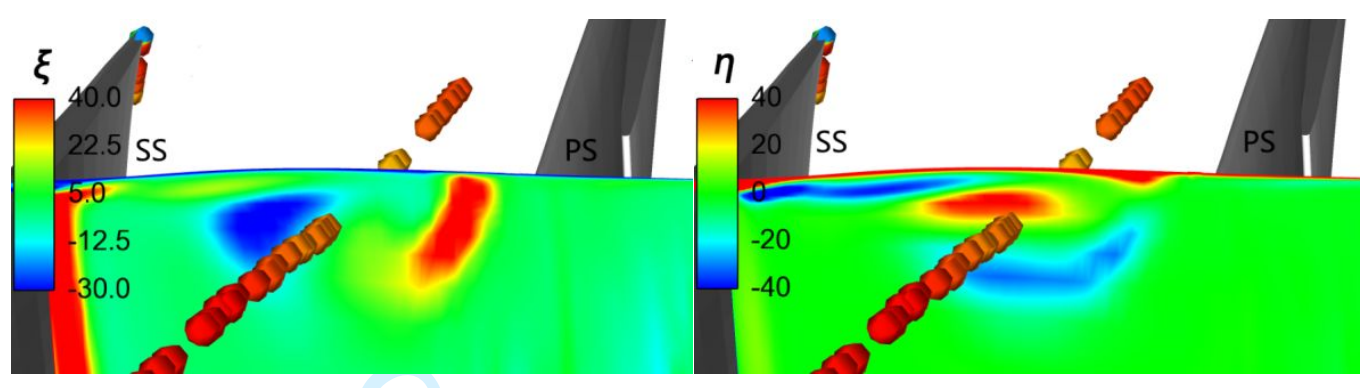

(a)

(b)

Figure 12 Normalised Radial (a) and circumferential vorticity (b) distributions where the vortex region expands at operating condition $\mathrm{U} 4$

In order to have a better understanding of the vortex expansion region, Fig. 12 presents circumferential and radial vorticity distributions at a cross section after the shock where region of vorticity expands to become more extensive. In Fig. 12a, it is found that the vortex core is surrounded by two regions of concentrated radial vorticity. The one nearest to the pressure side has positive radial vorticity, while the other one near the suction side is a negative radial vorticity region. A similar situation is also found in Fig. 12b where two regions of concentrated circumferential vorticity can be observed located above and below the vortex core. The analysis presented reveals that this region of expanding vorticity is characterised by a combination of radial and circumferential vorticity. Combined with the observations from Fig. 11, it is clear that the interaction between the TLV and the shock wave leads to a decrease of the streamwise component of vorticity, associated with a 
redistribution of the streamwise vorticity to radial and circumferential components (vorticity transport equation).

In summary, the interaction between the TLV and the shock wave induces an expansion of the vortex core due to the increase of the radial component of velocity and a redistribution of vorticity from the streamwise to the radial and circumferential components. Moreover, the vorticity redistribution region extends and gradually moves towards the PS of the adjacent blade when the compressor flow rate is reduced towards the stability limit.

\subsection{Flow details after the appearance of flow unsteadiness}

\subsubsection{Underlying mechanism for the inception of flow unsteadiness}

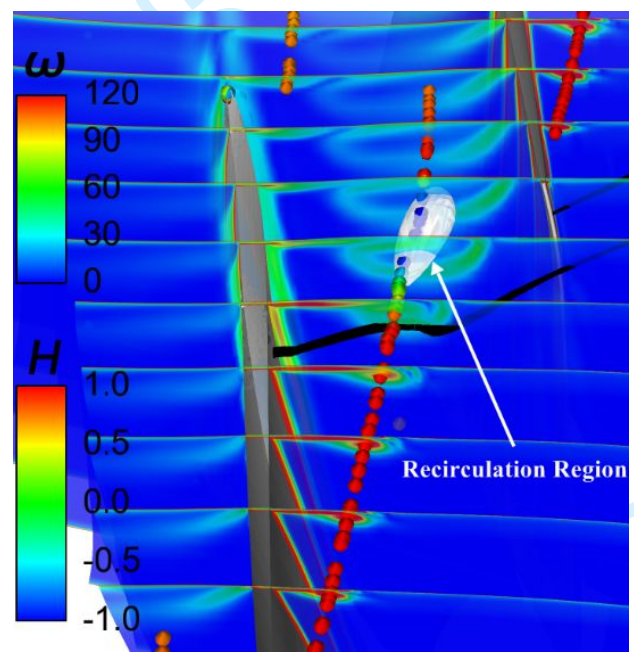

Figure 13 Normalized absolute vorticity distributions on cross-channel planes and

TLV core coloured with helicity at operating condition U5

As is shown in Fig. 13, flow features that have already been observed in the tip region at U4 such as vortex expansion and the disappearance of the vorticity concentrated region (see Fig. 10b) are also observed in U5. However, the blue part of the vortex core and the iso-volume identified by relative streamwise velocities less than $0 \mathrm{~m} / \mathrm{s}$ indicate that a 
stagnation point along with a recirculation region occur in U5. The presence of the sudden expansion of the vortex core, the disappearance of the concentrated region of absolute vorticity and the appearance of reverse flow near the vortex centreline implies that TLV breakdown occurs at the U5 condition, according to the criteria proposed by Sarpkaya [25], Leibovich [26] and Delery [27].

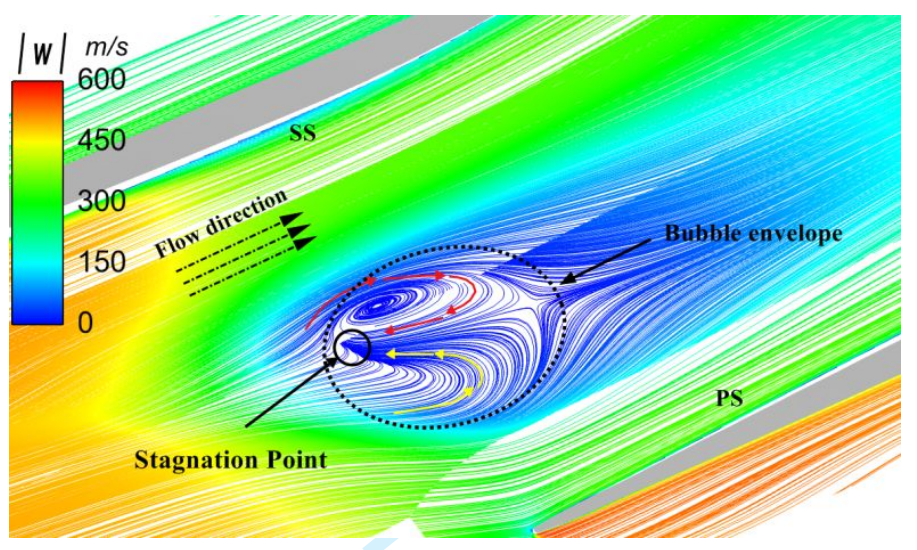

Figure 14 Instantaneous streamlines of relative velocity magnitude at $96 \%$ span at operating condition U5

In order to clarify which category of TLV breakdown occurs, Fig. 14 shows streamlines on the blade-to-blade plane through the vortex core (at $96 \%$ span). The streamlines are coloured with the absolute value of relative velocity. As is shown, firstly, the vortex axis is straight and parallel to the centreline. Secondly, two counter-rotating vortical structures appear that are almost symmetrical with respect to the centreline. It is therefore apparent that the TLV experiences a bubble-type breakdown [25-26]. As seen from the streamlines, the interaction between the main flow and reverse flow gives rise to an outer shape visualized as a bubble and a stagnation point which is located directly ahead of the recirculation region. The stagnation point along with the bubble works as a barrier 
to the incoming flow, resulting in a severe blockage in the passage and forcing the fluid outside the breakdown region to flow around the outer envelope of the bubble.

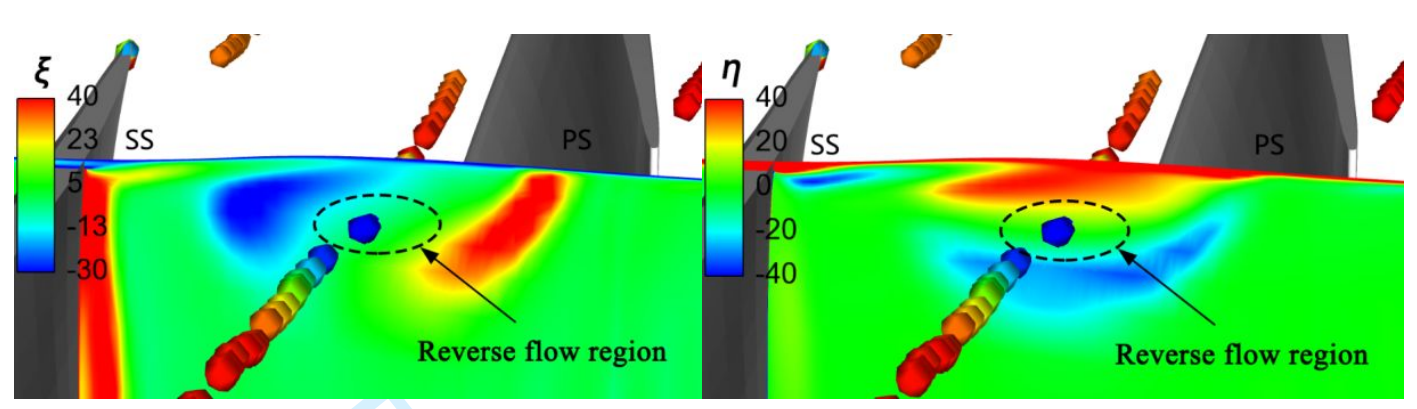

(a)

(b)

Figure 15 (a) Circumferential and (b) radial components of vorticity distributions where the vortex region expands at operating condition U5

Figure 15 presents the circumferential and radial components of vorticity distributions in the middle of the TLV breakdown region. Similar to that at condition U4, the breakdown region is also characterised by a combination of circumferential and radial component of vorticity. However, within the recirculation region, the concentrated regions of circumferential and radial vorticity can be regarded as a vortex ring structure that encircles the reverse flow region, and the streamlines on blade-to-blade plane shown in Fig. 14 can be interpreted as a cross-section of the three-dimensional structure. 


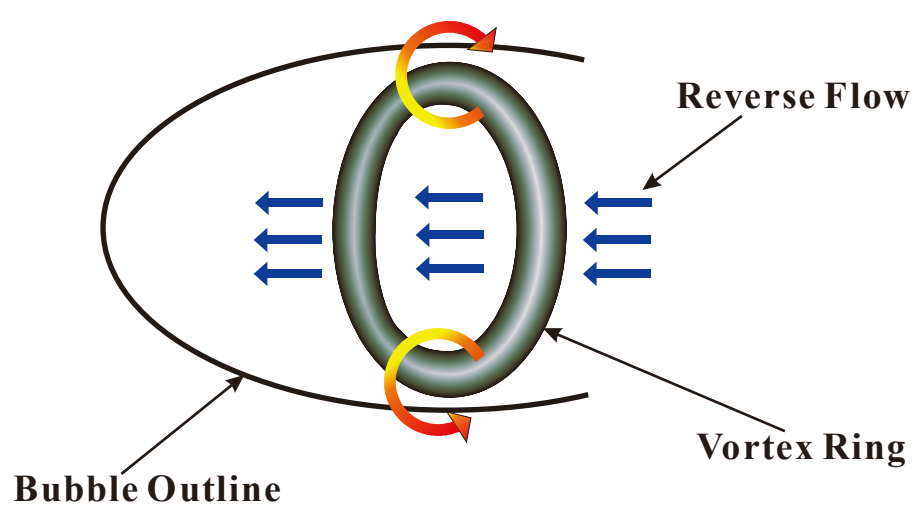

Figure 16 An illustration of the structure of the bubble-type vortex

In order to have a better understanding of the structure of the bubble-type vortex breakdown, especially the vortex ring, Fig. 16 provides a three dimensional illustration of the structure. As shown in this figure, the almost axisymmetric vortex ring, which is embedded in the outer part of the bubble, accounts for the transport of the mass flux in the bubble. The whole bubble system is filled from the aft portion of the bubble and emptied from the fore portion of the bubble, which is consistent with the observations reported from water tunnel experiments [25-27].

In the previous section, it was noted that there exists a redistribution of vorticity from the streamwise to the radial and circumferential components. At operating condition U5, the increase of radial and circumferential vorticity components strengthens the reverse flow at a lower radius. Then the radial velocity is thus further increased, which leads to an enhancement of the radial and circumferential vorticity components (conservation of angular momentum and vorticity transport equation). As a result, the reverse flow is again strengthened. This is a positive feedback mechanism through which the vortex ring structure gradually grows infinitely in radius. In order to achieve an equilibrium state of the whole system, a counteracting mechanism is need. The physical mechanism of the counteracting feedback can be described as follows: the acceleration of the reverse flow 
induces an increase of the radial velocity and further enhance the radial and circumferential vorticity components. On the other hand, the enhancement of the radial and circumferential vorticity components weakens the adverse pressure gradient at the upper radius, thus resulting in a deceleration of the reverse flow.

The whole system can be regarded as self-sustaining. These two feedback mechanism work alternatively, causing a periodical swelling and contraction of the bubble.

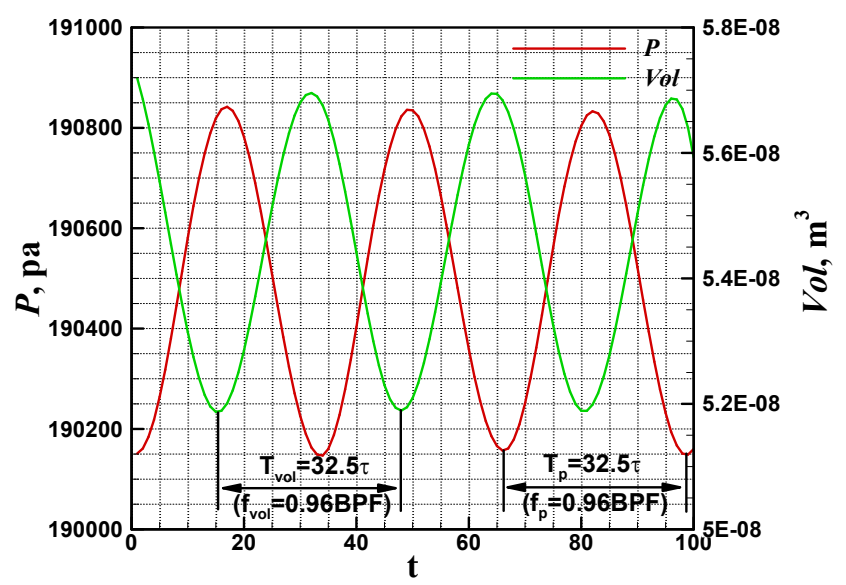

Figure 16 A comparison between the pressure fluctuation at $5 \% C_{a x}, 98.8 \%$ span and the variation of the recirculation region at operating condition U5

In order to make a quantitative study the fluctuation of the bubble, the variation of the volume of the recirculation region, which can be interpreted as representing the size of the bubble, is presented in Fig. 16. The volume of the recirculation region is defined as the dimension of the iso-volume identified by relative streamwise velocity less than $0 \mathrm{~m} / \mathrm{s}$. It is found that the volume of the recirculation region fluctuates periodically with time. The period is $32.5 \tau$, which corresponds to the characteristic frequency of the flow field of 0.96 BPF that was noted earlier for this operating condition. That is to say, the fluctuation of the bubble size is closely related to the unsteadiness of the flow field. Moreover, there exists a negative correlation between the static pressure at the LE of the blade and the volume of 
the recirculation region: when the volume of the recirculation region increases, the static pressure will decrease and vice versa. Therefore, it can be proposed that the fluctuation of the bubble dominates the flow unsteadiness by determining the static pressure distribution at the blade tip. Namely, when the bubble swells, it blocks the passage and induces a decrease of the static pressure near the pressure side of blade tip; when the bubble shrinks, the static pressure recovers.

Comparing with the flow field in U4 where no vortex breakdown nor flow unsteadiness is found, it can be concluded that reverse flow is a necessary condition for vortex breakdown. Moreover, it plays a key role in both positive and counteracting feedback process which dominating the periodical fluctuation of the bubble.

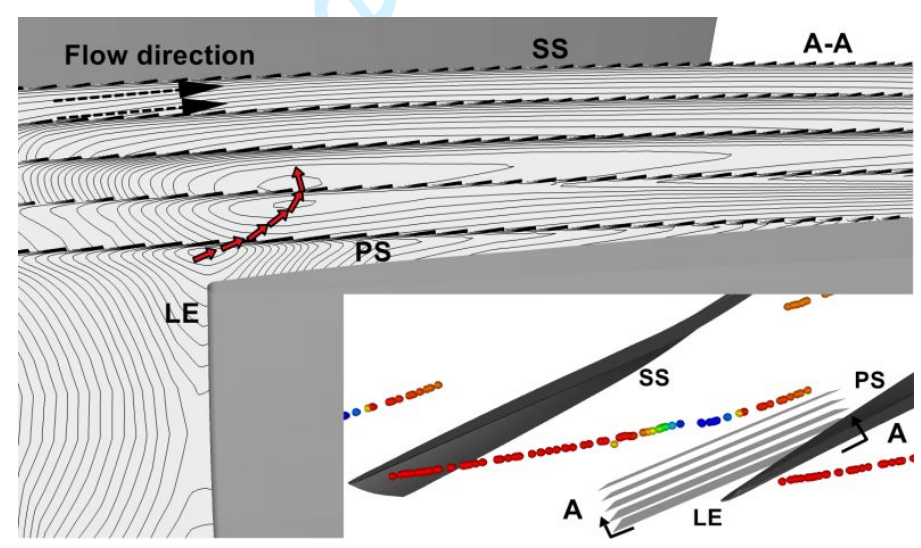

Figure 17 Contours of constant relative velocity near the blade tip at U5

Considering that the TLV is situated in the blade tip region near the casing, the influence of the horseshoe vortex should be taken into consideration. Figure 17 presents contours of constant relative velocity near the blade tip. Note that contour values lie in the range from 0 to $500 \mathrm{~m} / \mathrm{s}$ in $50 \mathrm{~m} / \mathrm{s}$ intervals. The trajectory of the PS leg of the horseshoe vortex is marked with red arrows. As can be seen, the pressure leg of the horseshoe vortex extends from the LE of the blade to the middle part of the passage with a direction nearly 
perpendicular to the PS of the blade, and finally it dissipates before encountering the TLV. Consequently, it seems that no horseshoe vortex/TLV interaction occurs at this operating condition.

\subsubsection{Underlying mechanism for the change of characteristic frequency and amplitude of flow unsteadiness}

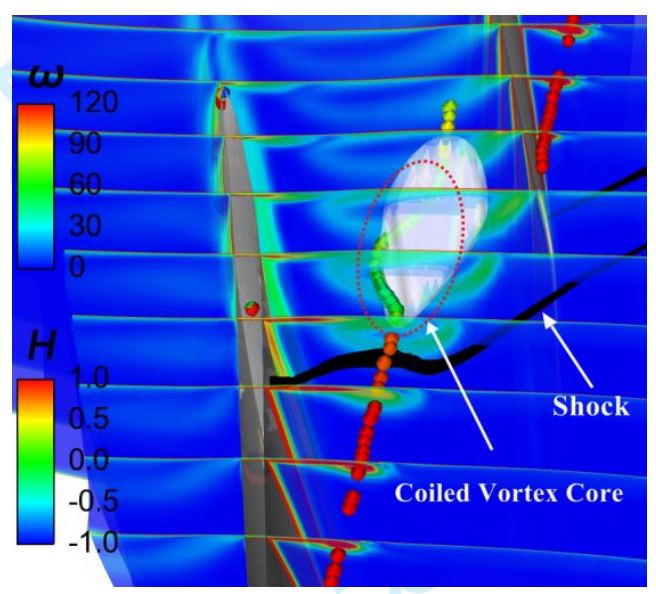

Figure 18 Normalized absolute vorticity distributions on cross-channel planes and TLV core coloured with helicity at the U7 condition

Figure 18 shows the time-averaged tip flow field at the U7 condition. It is found that the TLV also breaks down at this operating point, since a vortex expansion, a disappearance of the concentrated region of vorticity and a large-scale recirculation region are clearly shown. However, the vortex core is not straight forward. The vortex core line tilts away from the centreline, and twists and turns in the passage after interacting with the shock wave. It turns out that the two vortices situated on either side of the vortex axis are no longer symmetrical with respect to the centreline as is shown in Fig. 19. All these features correspond to the characteristics of spiral-type vortex breakdown proposed by Leibovich 
[26] and Brücker [28]. It is thus shown that the TLV experiences a spiral-type breakdown at this operating condition.

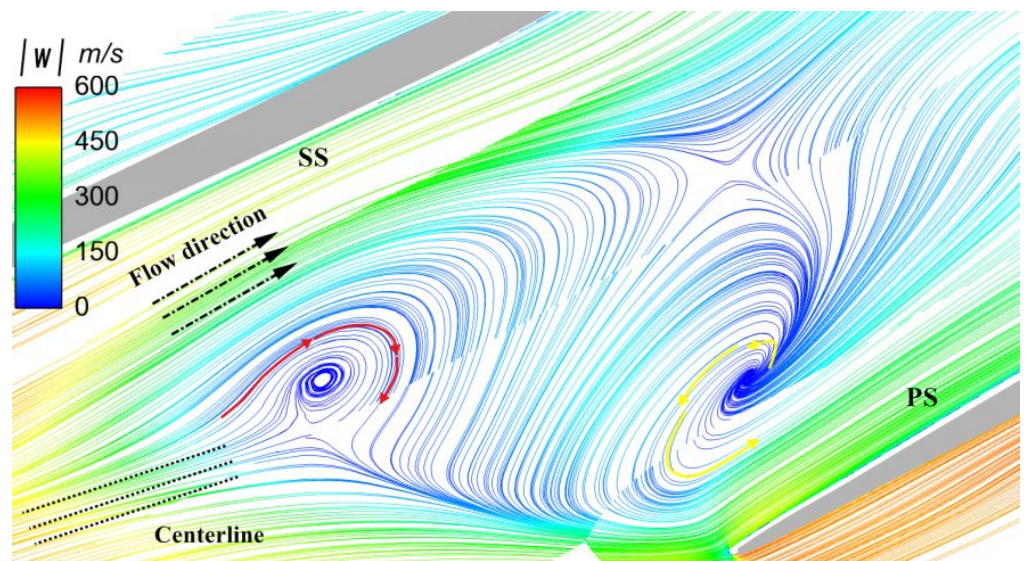

Figure 19 Instantaneous streamlines of relative velocity magnitude at $96 \%$ span at operating condition U7
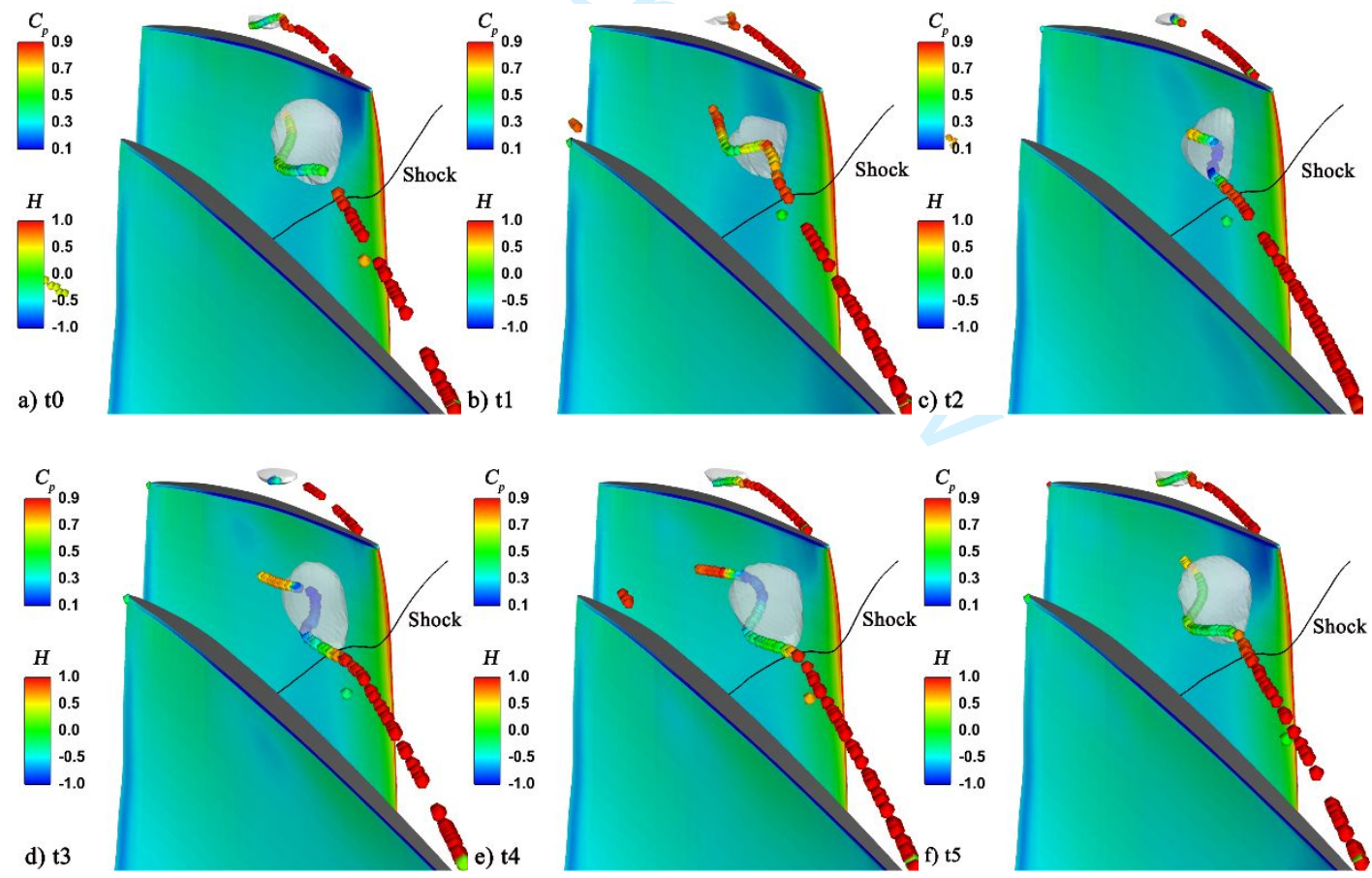

Figure 20 Instantaneous flow structures and $C_{p}$ distributions on the pressure surface during one period at $\mathrm{U} 7$ 
Figure 20 presents the flow structure and the static pressure coefficient distribution during one period. The static pressure coefficient is given by:

$$
C_{p}=\frac{P-P_{r e f}}{0.5 * \rho U^{2}}
$$

Where $P_{\text {ref }}$ is the reference static pressure, $\rho$ is the density, $U$ is the blade tip speed.

It is observed that flow structure in Fig. 20f is similar to that in Fig. 20a, which is designated as the beginning of the period. It is also found that the coiled vortex core rotates around the centerline in the same sense as the tip leakage flow, whereas its sense of winding is opposite to it. Obviously, the rotation of the asymmetric vortex structure is a violent and unsteady flow phenomenon and will induce a severe blockage in the tip region. The duration of one period is estimated from these figures as $57 \tau$, corresponding to the characteristic frequency of $0.53 \mathrm{BPF}$ observed in the flow field at this operating condition in Fig. 6. Hence, it appears that the agitation of the spiral vortex core is one of the inducements to the unsteadiness of the whole system.

It is also observed in Fig. 20 that with the rotation of the vortex core, the recirculation region fluctuates with time. As noted in the section 3.4.1, the fluctuation of the recirculation region has a significant influence on the unsteadiness of the static pressure in the tip region on the PS of the blade. The $C_{p}$ contours in Fig. 20 show how, during the period from t0 to $\mathrm{t} 2$, the recirculation region gradually shrinks, resulting in a recovery of the static pressure near the LE of the PS. Then during the period from $\mathrm{t} 3$ to $\mathrm{t} 5$, the recirculation region progressively grows, leading to a large-scale low pressure region on the PS. This process is similar to that described at the U5 condition. However, according to the FFT results in Fig. 8, the pressure fluctuation at the U7 condition is much more severe than that at the U5 
condition. In order to clarify this phenomenon, Fig. 21 compares the volumes of the recirculation region between the U7 and U5 conditions. It is found that the amplitude of fluctuation of the recirculation region at the U7 condition is approximately 14 times larger than that at the U5 condition. The much larger oscillation of the recirculation region leads to a more severe fluctuation of the static pressure near the LE of the PS. This explains why the amplitude of the pressure oscillation increases strongly in the transition from U5 condition to U7 condition in Fig. 6.

The period of the fluctuation of the recirculation region at $\mathrm{U} 7$ is $57 \tau$ in Fig. 21, which corresponds to $0.53 \mathrm{BPF}$ and is identical to the strongest characteristic frequency in the FFT spectrum of the flow field at this operating condition. It is thus inferred that the fluctuation of the recirculation region is also one of the inducements of the unsteadiness of the whole system.

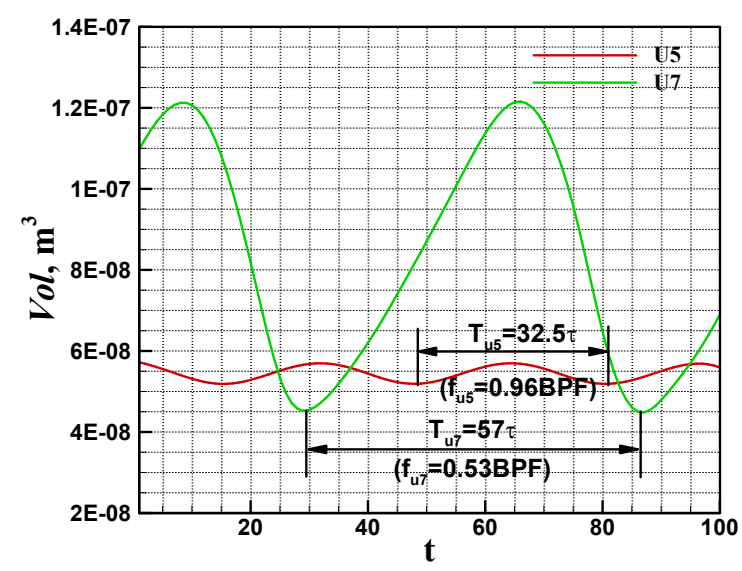

Figure 21 A comparison of the volumes of the recirculation region between U5 and $\mathrm{U} 7$

Since the curved vortex core is rotating, when it turns to the pressure side, the interaction between the TLV and the pressure side leg of the horseshoe vortex should be 
taken into consideration. Figure 22 shows contours of constant relative velocity near the blade tip at U7. The pressure side leg of the horseshoe vortex extends to the middle part of the passage and interacts with the TLV, which is different from the situation at U5 condition in Fig. 17.

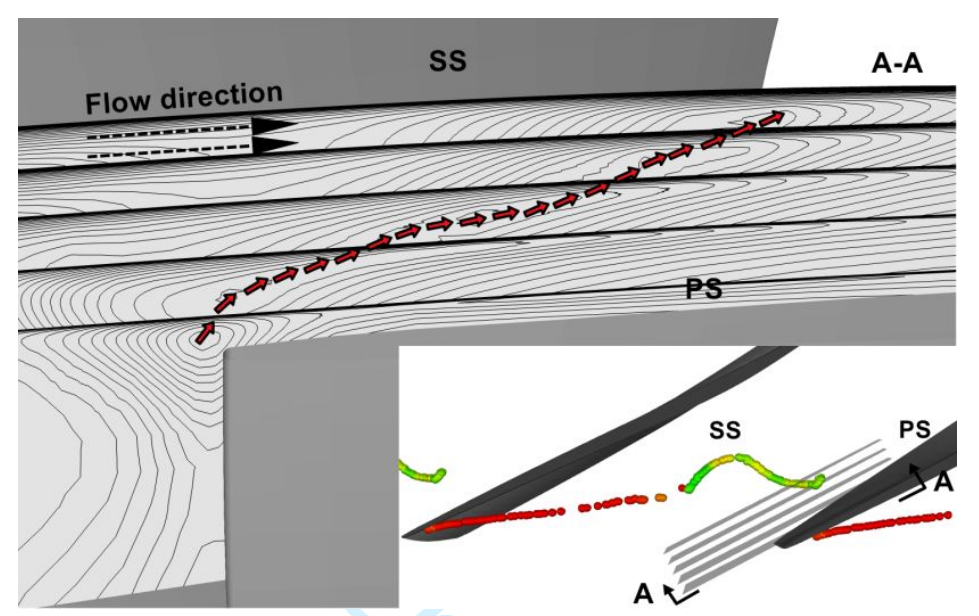

Figure 22 Contours of constant relative velocity near the blade tip at U7

Figure 23 shows a schematic view of the interaction between the horseshoe vortex and the TLV. As is shown, the TLV has a positive axial vorticity, whereas the pressure side leg of the horseshoe vortex has a negative axial vorticity. When the TLV core turns towards the PS of the passage, the intensity of both vortices is weakened due to the interaction between these two vortices, which further leads to an increase of the static pressure on the PS (see Fig. 20a-20d). When the TLV core turns towards the SS, these two vortices will no longer interact with each other, resulting in a recovery of the vortex intensity and further a decrease of the static pressure on the PS (see Fig. 20d-20f). 


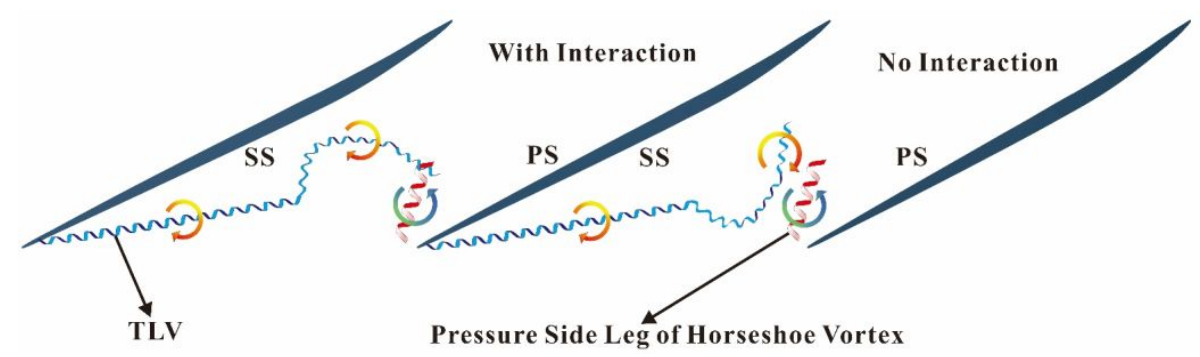

Figure 23 A schematic view of the interaction between the horseshoe vortex and the TLV

To conclude, the TLV experiences a spiral-type breakdown at the U7 condition. The transition of vortex breakdown form from bubble-type to spiral-type is considered as the main cause of the significant reduction in the characteristic frequency of the pressure oscillation as is shown in the FFT results. Furthermore, there are in total three mechanisms that lead to flow unsteadiness at this operating condition. The first two mechanisms can be regarded as inherent characteristics of the spiral-type vortex breakdown, namely the rotating of the coiled vortex core and the fluctuation of the recirculation region. The last one is the interaction between the vortex core and the pressure side leg of the horseshoe vortex. The joint action of these three mechanisms provokes the flow unsteadiness in this operating condition. As for the jump of the amplitude of the pressure oscillation, it can be attributed to the severe fluctuation of the recirculation region at U7 and the joint action of the above mentioned three mechanisms which provoke the flow unsteadiness at this operating condition. It is also inferred that the frequency of the flow field unsteadiness does not solely depend on the frequency of the vortex breakdown, but depends on the whole flow properties in the confined rotor-passage system with the leakage flow. 


\section{CONCLUSIONS}

In this paper, a series of URANS simulations have been conducted on NASA Rotor 35 , and the results were validated by experimental data. The unsteady behaviours of the test rotor have been studied in detail. Then the tip flow structures, especially the TLV breakdown, have been investigated to shed lights on the origin and underlying mechanisms of the flow unsteadiness. The results can be summarized as follows:

(1) Analyses of monitoring results of numerical probes showed that three typical flow characteristics occurred as the operating condition approached the stability limit: no mass flow oscillation was detected before operating condition U5; mass flow oscillations appear at U5 and U6 with a characteristic frequency approximately equal to the BPF; mass flow oscillations appear from U7 to U10 with a larger amplitude and a lower characteristic frequency of approximately half of the BPF.

(2) Further analyses of the tip flow field showed that the evolution of the TLV experienced three different stages. At the first stage, the TLV did not breakdown, but the shock/vortex interaction gave rise to an expansion of the vortex core due to the increased radial component of velocity and a redistribution of vorticity from the axial to radial and circumferential components. At the second stage, the TLV experienced a bubble-type breakdown. An alternation of positive and counteracting feedback resulted in a periodic swelling and contraction of the bubble, which was thought to be the cause of the pressure fluctuation $(0.96 \mathrm{BPF})$ at the blade tip. At the third stage, the TLV breakdown form transitioned from bubble-type to spiral-type. A joint action of three mechanisms (the rotation of the vortex core, the fluctuation of the recirculation region 
and the interaction between the TLV and the pressure side leg of the horseshoe vortex) contributed to strong pressure fluctuation (0.53BPF) at this operating condition.

(3) The shock/vortex interaction did not directly induce a vortex breakdown, but it led to an expansion of the vortex core and a redistribution of vorticity from axial to radial and circumferential components.

(4) The existence of reverse flow is a necessary condition for bubble-type breakdown of the TLV since it played an important role in both the positive and counteracting feedback processes which drove the periodic fluctuation of the bubble.

\section{ACKNOWLEDGMENTS}

The authors would like to acknowledge the supports of National Natural Science Foundation of China, Grant No. 11572257, 51790512, 51536006, and the Innovation Foundation for Doctor Dissertation of Northwestern Polytechnical University No. CX201911.

\section{DECLARATIONS OF INTEREST}

The authors declare no conflict of interest.

\section{REFERENCES}

[1] Denton JD. Loss Mechanisms in Turbomachines. J Turbomach 2008;115:621. doi:10.1115/1.2929299.

[2] Adamczyk JJ, Celestina ML, Greitzer EM. The Role of Tip Clearance in High-Speed Fan Stall. J Turbomach 1993;115:28. doi:10.1115/1.2929212. 
[3] Zhang L, Zhang L, Zhang Q, Jiang K, Tie Y, Wang S, et al. Effects of the SecondStage of Rotor with Single Abnormal Blade Angle on Rotating Stall of a Two-Stage Variable Pitch Axial Fan. Energies 2018;11:3293. doi:10.3390/en11123293.

[4] Inoue M, Kuroumaru M, Fukuhara M. Behavior of Tip Leakage Flow Behind an Axial Compressor Rotor. J Eng Gas Turbines Power 1986;108:7. doi:10.1115/1.3239889.

[5] Kameier F, Neise W. Experimental Study of Tip Clearance Losses and Noise in Axial Turbomachines and Their Reduction. J Turbomach 1997;119:460. doi:10.1115/1.2841145.

[6] Holzinger F, Wartzek F, Jüngst M, Schiffer H-P, Leichtfuss S. Self-Excited Blade Vibration Experimentally Investigated in Transonic Compressors: Rotating Instabilities and Flutter. J Turbomach 2016;138:041006. doi:10.1115/1.4032163.

[7] Schlechtriem S, Lötzerich M. Breakdown of Tip Leakage Vortices in Compressors at Flow Conditions Close to Stall. Vol. 1 Aircr. Engine; Mar. Turbomachinery; Microturbines Small Turbomach., ASME; 1997, p. V001T03A004. doi:10.1115/97GT-041.

[8] Furukawa M, Inoue M, Saiki K, Yamada K. The Role of Tip Leakage Vortex Breakdown in Compressor Rotor Aerodynamics. J Turbomach 1999;121:469. doi:10.1115/1.2841339.

[9] Furukawa M, Saiki K, Yamada K, Inoue M. Unsteady Flow Behavior due to Breakdown of Tip Leakage Vortex in an Axial Compressor Rotor at Near-Stall 
Condition. Vol. 1 Aircr. Engine; Mar. Turbomachinery; Microturbines Small Turbomach., ASME; 2000, p. V001T03A112. doi:10.1115/2000-GT-0666.

[10] Yamada K, Furukawa M, Nakano T, Inoue M, Funazaki K. Unsteady ThreeDimensional Flow Phenomena Due to Breakdown of Tip Leakage Vortex in a Transonic Axial Compressor Rotor. Vol. 5 Turbo Expo 2004, Parts A B, ASME; 2004, p. 515-26. doi:10.1115/GT2004-53745.

[11] Yamada K, Funazaki K, Sasaki H. Numerical Investigation of Relation Between Unsteady Behavior of Tip Leakage Vortex and Rotating Disturbance in a Transonic Axial Compressor Rotor. Vol. 6 Turbomachinery, Parts A, B, C, ASME; 2008, p. 42536. doi:10.1115/GT2008-50779.

[12] Hah C, Rabe DC, Wadia AR. Role of Tip-Leakage Vortices and Passage Shock in Stall Inception in a Swept Transonic Compressor Rotor. Vol. 5 Turbo Expo 2004, Parts A B, ASME; 2004, p. 545-55. doi:10.1115/GT2004-53867.

[13] Bergner J, Kinzel M, Schiffer H-P, Hah C. Short Length-Scale Rotating Stall Inception in a Transonic Axial Compressor: Experimental Investigation. Vol. 6 Turbomachinery, Parts A B, vol. 2006, ASME; 2006, p. 131-40. doi:10.1115/GT2006-90209.

[14] Zhang H, Deng X, Chen J, Huang W. Unsteady tip clearance flow in an isolated axial compressor rotor. J Therm Sci 2005;14:211-9. doi:10.1007/s11630-005-0004-4. 
[15] Zhang H, Deng X, Lin F, Chen J, Huang W. A Study on the Mechanism of Tip Leakage Flow Unsteadiness in an Isolated Compressor Rotor. Vol. 6 Turbomachinery, Parts A B, vol. 2006, ASME; 2006, p. 435-45. doi:10.1115/GT2006-91123.

[16] Tong Z, Lin F, Chen J, Nie C. The Self-Induced Unsteadiness of Tip Leakage Vortex and Its Effect on Compressor Stall Inception. Vol. 6 Turbo Expo 2007, Parts A B, ASME; 2007, p. 1551-62. doi:10.1115/GT2007-27010.

[17] Du J, Lin F, Zhang H, Chen J. Numerical Investigation on the Originating Mechanism of Unsteadiness in Tip Leakage Flow for a Transonic Fan Rotor. Vol. 6 Turbomachinery, Parts A, B, C, ASME; 2008, p. 707-17. doi:10.1115/GT2008-51463.

[18] Wu Y, Li Q, Chu W, Zhang H, Su Z. Numerical investigation of the unsteady behaviour of tip clearance flow and its possible link to stall inception. Proc Inst Mech Eng Part A J Power Energy 2010;224:85-96. doi:10.1243/09576509JPE793.

[19] Wu Y, Li Q, Tian J, Chu W. Investigation of Pre-Stall Behavior in an Axial Compressor Rotor-Part I: Unsteadiness of Tip Clearance Flow. J Turbomach 2012;134:051027. doi:10.1115/1.4004752.

[20] Reid L, Moore R D. Design and Overall Performance of Four Highly-Loaded, High Speed Inlet Stages for an Advanced, High Pressure Ratio Core Compressor. NASA TP-1337,1978.

[21] Van Zante DE, Strazisar AJ, Wood JR, Hathaway MD, Okiishi TH. Recommendations for Achieving Accurate Numerical Simulation of Tip Clearance 
Flows in Transonic Compressor Rotors. J Turbomach 2000;122:733. doi:10.1115/1.1314609.

[22] Yanhui $\mathrm{Wu}$, Guangyao An. Numerical Investigation into the Unsteady Flow and its Associated Flow Mechanism in a Transonic Compressor Rotor at Near Stall Conditions. Journal of Propulsion Technology, 37(10), 2016. doi:10.13675/j.cnki.tjjs.2016.10.006.

[23] JAMESON A. Time dependent calculations using multigrid, with applications to unsteady flows past airfoils and wings. 10th Comput. Fluid Dyn. Conf., Reston, Virigina: American Institute of Aeronautics and Astronautics; 1991. doi:10.2514/6.1991-1596.

[24] Sujudi D, Haimes R. Identification of swirling flow in 3-D vector fields. 12th Comput. Fluid Dyn. Conf., Reston, Virigina: American Institute of Aeronautics and Astronautics; 1995. doi:10.2514/6.1995-1715.

[25] Sarpkaya T. On stationary and travelling vortex breakdowns. J Fluid Mech 1971;45:545-59. doi:10.1017/S0022112071000181.

[26] Leibovich S. The Structure of Vortex Breakdown. Annu Rev Fluid Mech 1978;10:221-46. doi:10.1146/annurev.fl.10.010178.001253.

[27] Delery JM. Aspects of vortex breakdown. Prog Aerosp Sci 1994;30:1-59. doi:10.1016/0376-0421(94)90002-7.

[28] Brücker C. Study of vortex breakdown by particle tracking velocimetry (PTV). Exp Fluids 1993;14:133-9. doi:10.1007/BF00196996. 